\title{
Téoros
}

Revue de recherche en tourisme

\section{Les défricheurs du ciel!}

\section{L'histoire des pionniers de l'air du Québec}

\section{Jeanne Poulin}

Volume 14, numéro 2, été 1995

Le tourisme : toute une histoire!

URI : https://id.erudit.org/iderudit/1075099ar

DOI : https://doi.org/10.7202/1075099ar

Aller au sommaire du numéro

Éditeur(s)

Université du Québec à Montréal

ISSN

0712-8657 (imprimé)

1923-2705 (numérique)

Découvrir la revue

Citer cet article

Poulin, J. (1995). Les défricheurs du ciel! L'histoire des pionniers de l'air du Québec. Téoros, 14(2), 30-31. https://doi.org/10.7202/1075099ar d'utilisation que vous pouvez consulter en ligne.

https://apropos.erudit.org/fr/usagers/politique-dutilisation/ 


\title{
Les défricheurs du ciel! \\ L'histoire des pionniers de l'air du Québec
}

\author{
Jeanne Poulin *
}

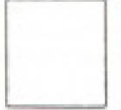

Il faut sans cesse rappeler que notre gloire a aussi des ailes, que notre histoire est autant peuplée d'aviateursetdemécaniciens que d'artistes et d'artisans, que notre culture s'est autant enrichie par le ronronnement des moteurs d'avions que par le lyrisme de nos chansons, et enfin, que notre langue a été façonnée par nos gens de l'air comme par nos législateurs, nos écrivains et nos traducteurs.

Une passion sans borne, presque inexplicable, animait nos défricheurs du ciel! Une mission spirituelle les poussait vers ce nouvel horizon, parcouru au tournant du siècle par des esprits fiers, des héros en quête de liberté et de défis.

Imaginez nos premiers aviateurs, qui, du haut de leurs quelque mille pieds, admirent pour la première fois des lacs ondulés par les glaces et la neige, des forêts à perte de vue, et parfois, lorsque les dieux de la faune veillaient sur eux, des troupeaux de caribous, de chevreuils et de loups.

Imaginez certeépoque lointaine quel'aviation a chambardée, y compris toutes nos vieilles perceptions et nos vieilles idées.

Aujourd'hui encore, on ne connaît rien sur l'histoire de l'aviation au Québec, rien sur ses découvreurs, ses militaires, ses écrivains, ses ingénieurs et ses mécaniciens: les Jean-Marie Landry, Roméo Vachon, Arthur Fecteau, Stuart Graham et Roger Demers, pour nommer que ceux-ci.

Pourtant, on connait les frères Wright, qui ont accompli le premier vol motorisé au monde, et John McCurdy qui a réussi le premier vol au Canada. Mais les aviateurs de chez nous, eux, restent dans l'oubli.

\section{Des pionniers mal connus}

L'aviation au Québec a connu ses débuts au Lac-à-la-Tortue, considéré aujourd'hui comme étant le berceau de l'aviation com-

Madame Jeanne Poulin est journaliste en matieres socio-culturelies et directrice des communications de la Fondation Adrovision Quebec. merciale au Canada. On comptait 17 pilotes et mécaniciens sur ce site, où on y construisait et réparait des appareils. A l'époque, la Laurentide Pulp and Paper Co., l'utilisa pour la surveillance contre les feux de forêts et pour la photographie aérienne. Deux hydravions formèrent, en 1919 , le noyau de l'aviation commerciale au Canada. Presque tous les pionniers de l'air du Québec ont amerri sur ce lac devenu célèbre.

Jean-Marie Landry, cel ui quel'on considère commele premier pilotecanadien-français, s'est rendu à l'école de Louis Blériot en France en 1914 où il a obtenu son brevet. II fit quelques vols au-dessus des champs de bataille européens avant de revenir au Québec. Féru d'acrobatie aérienne, il s'est aventuré sous le pont de Québec et fit la manchette.

Roméo Vachon, connu comme étant le premier facteur volant, assura la livraison du courrier en 1927 à partir de l'embouchure du fjorddu Saguenayjusqu'au détroit de Belle-Isle. C'était le début de l'aéropostale pour cutte région éloignée. A maintes reprises, Roméo Vachon transporta les malades vers les grands centres, devenant ainsi le premier ambulancier volant. Le comté de Vachon a été baptisé en son hommage. Il fut le premier directeur de l'aćroport de Saint-Hubert, premier aéroport commercial au Canada.

Arthur Fecteau, suite à son cours de pilotage à Québec, fut le premier francophone à exiger de recevoir son brevet de pilote en français de la Défense nationaleoùl'anglais était la seule langue connue. Il est parta pour l'Abitibi en 1930 aux commandes d'un vieux biplan et forma Fecteau Air Service, qui fut considéré après la guerre comme une des plus importantes compagnies d'aviation de brousse au monde. Durant les années 1950 , ses pilotes ont transporté à bord de leurs hydravions de type Beaver les arpenteurs qui ont jaugé les lacs et les rivières se déversant dans la Baie James, contribuant ainsi au développement des barrages électriques d'Hydro-Québec.

Quant à Stuart Graham, le premier pilote de brousse au monde, il patrouilla, à bord de la Vigilance, le ciel du Québec pour la détection des feux de forêts, participa aux inventaires forestiers en Mauricie et conuribua à la réalisation des premières cartes par photographies aériennes. Cet aviateur courageux est également connu pour avoir diffusé, du haut des airs, des dépliants bilingues incitant le peuple à protéger ses forêts. Sa carrière débuta en 1919.
Jean-Marie Landry, premier pilote francophone du Québec, formé à l'école de Louis Blériot en France en 1914.

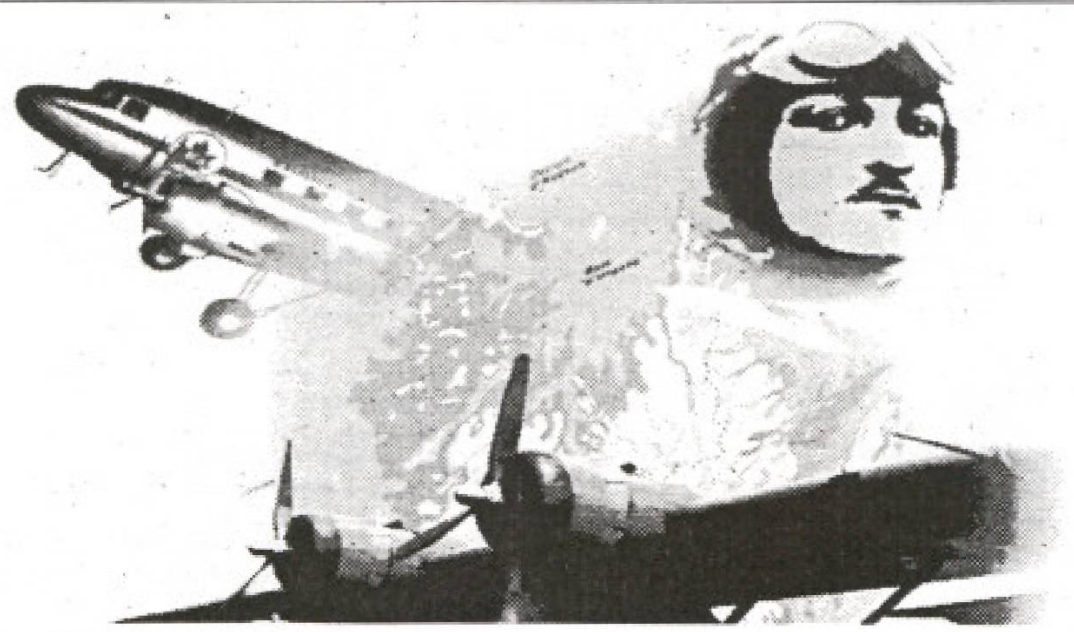




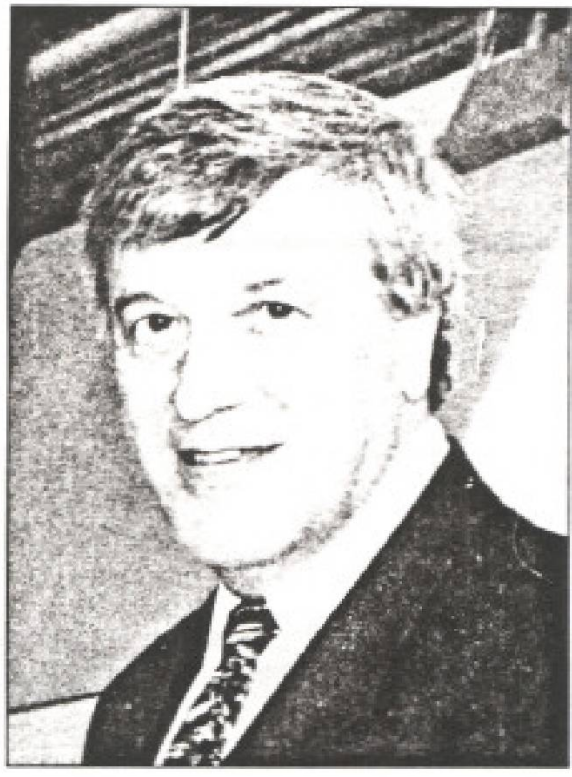

Le commandant Michel St-Amaud, président de la Fondation Aćrovision Québec.

Si ces aviateurs et leurs exploits ne font pas partie de notre mémoire collective, peutêtre se souvient-on d'un exploit qui al largement contribué à l'affirmation linguistique de la société québécoise. Il s'agit de la lutte pour le français dans l'air, bataille remportée au début des années 1970 par les pilotes francophones regroupés dans l'Association des gens de l'air du Québec. C'est grâce à la ténacité et aux convictions de Roger Demers et Jean-Guy Carrière, entre autres, qu'on a prouvé qu'il n'était ni honteux ni insécuritaire de parler la langue française dans le ciel du Québec.

\section{Un musée en gestation.:}

Le Musée de l'aviation du Québec veut inscrire les noms des premiers aviateurs québécois à notre mémoire collective en proposant un temple qui leur donnera la gloire qu'ils méritent.

Àchaque jour, le Québec perd des aéronefs rares et précieux: les Beaver, Otter, Norseman et Husky, entre autres. Il est donc devenu urgent de récupérer, de restaurer etd'entreposer les appareils, artefacts, documents et photographies qui ont marqué l'histoire de l'aviation québécoise.

Incorporée en janvier 1991, la Fondation Aérovision Québec, promoteur du Musée de l'aviation du Québec, regroupe aujourd'hui 200 membres qui, ensemble, forment un puissant groupe de pression. Pilotes, mécaniciens, industriels, militai- res, historiens, collectionneurs, artistes, écrivains et élus travaillent ensemble depuis 4 ans à concrétiser ce projet.

Mais, aujourd'hui, les muséesdoiventassumer une grande part de leurs frais. Pour survivre, il faut donc s'autofinancer. On note d'ailleurs une augmentation des boutiques de souvenirs et des restaurants au sein même des établissements muséaux.

La mise en valeur du contenu émotionnel de l'expérience culturelle et touristique étant maintenant acceptée en gestion muséale, les musées de l'aviation peuvent organiser des spectacles, des meetings aériens ou d'autres attractions afin de financer une partie de leurs opérations. Aux États-Unis, on compte des milliers d'avions privés et une longue tradition en aćrotourisme. De nombreux petits aéroports se sont dotés d'infrastructures qui contribuent aux revenus de leur région par l'organisation d'activités qui attirent les aérotouristes et le grand public. Monsieur St-Arnaud, organisateur d'événements aéronautiques à grand déploiement, désire exploiter ce marché de prestige à l'aéroport de Saint-Hubert. Selon une étude effectuée par un groupe d'étudiants de maitrise en gestion de projets de l'UQAM pour le compte de la Fondation, la tenue d'un événement aéronautique à Saint-Hubert ferait de son aéroport un lieu de pèlerinage annuel pour l'aviateur en quêtre d'aventures. Toujours selon cette étude, une activité bien préparé̃e attirera autant les aérotouristes étrangers que le grand public québécois et générera d'importantes retombées économiques pour lesintervenants touristiques situés en Montérégie et dans la grande région métropolitaine.

Cet événement ressemblera peut-êtrre à celui d'Oshkosh, dans le Wisconsin, considéré comme La Mecque de l'aviation pri-

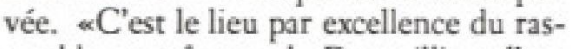
semblement fraternel. Des milliers d'appareils et des centaines de milliers de visiteurs manifestent leur passion pour l'aviations, d'expliquer monsieur St-Arnaud qui souhaite mettre sur pied un mini-Oshkosh à Saint-Hubert.

En plus de préparer un événement touristique populaire, les organisateurs devront réaliser un vếritable tour de force: il faudra que le Musée de l'aviation du Québec soit un pôle d'attraction séduisant. Pour réussir ce coup, les promoteurs du Musée devront offrir un produit culturel à la hauteur de la concurrence. Rappelons qu'il existe 17 musées de l'aviation au Canada, des centaines aux Etats-Unis et pas un seul au Québec.

\section{L'aérotourisme: un tourisme de prestige inconnu au Québec}

Rodolphe Pagé a contribué à l'histoire québécoise de l'aviation durant les années 1930 pour avoir été le premier pilote québécois à construire son propre avion; mais, aujourd'hui encore, voler de ses propres ailes est un phénomène exceptionnel ici car peu de Québécois possèdent leur propre machine volante. Pourtant, cetteexpérience est enivrante. $*$ C'est vivre au sommet des émotions:, de dire le commandant St-Arnaud, président de la Fondation Aérovision Québec. Pilote chevronné avec 40 ans d'expérience et plus de 22000 heures de vol à son carnet, monsieur St-Arnaud s'inspire de la philosophie des grands écrivains-aviateurs pour expliquer l'attrait du ciel: $*$ Richard Bach, auteur de Jonathan Linvingston, le goéland a bịen décrit cette expérience spirituelle. A 10000 pieds d'altrtude, l'homme et sa machine ne forment qu'une entité, qui elle seule est capable de jouir d'une vision exaltante de l'univers. Selon Bach, cette fusion chasserait angoisse et mauvais espritsw. C'est tout dire!

Lorsque l'aviateur est perçu sous cet angle, on peut comprendre qu'il soit à la recherche constante du merveilleux. Privilégié par l'argent et la possibilité de choisir, ce typede touristeveutdécouvrir de nouvelles destinations lui permettant de renouveler ces sensations.

En un premier temps, le Québec invitera tous ces * fous» de l'air à venir démontrer ici tout le potentiel de l'aérotourisme. $f$

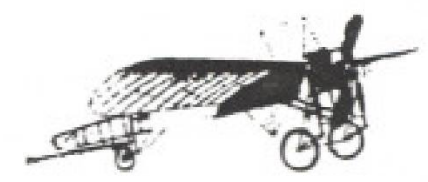

